\title{
Effect of Dexmedetomidine on The Neuroglobin Expression in Elderly Patients With Minimally Invasive Coronary Artery Bypass Graft Surgery
}

\author{
Yan Gao, ${ }^{1}$ Huidan Yu, ${ }^{2}$ Wenlong Wang, ${ }^{3}$ Yeming Wang,, Jinliang Teng, ${ }^{1}$ Fulong $\mathrm{Li}^{1}$ \\ ${ }^{1}$ Department of Anesthesiology, The First Affiliated Hospital of Hebei North University; ${ }^{2}$ Department of Ultrasound Medicine, The \\ First Affiliated Hospital of Hebei North University; ${ }^{3}$ Operating Room, The First Affiliated Hospital of Hebei North University, \\ Zhangjiakou, Hebei
}

\section{ABSTRACT}

Background: To study the effect of dexmedetomidine (Dex) on the expression of Neuroglobin (Ngb) and postoperative cognitive function in elderly patients undergoing minimally invasive coronary artery bypass surgery.

Methods: Forty patients, who underwent elective minimally invasive off-pump coronary artery bypass grafting in our hospital from January 2018 to December 2019, were randomly divided into the Dex group $(\mathrm{N}=20)$ and control group $(\mathrm{N}=20)$. Venous blood samples were taken to determine the expression level of $\mathrm{Ngb}$ in both groups. Mini mental status examination (MMSE) was used to detect the cognitive function of patients.

Results: The expression level of Ngb in the Dex group was significantly higher than that in the control group at $6 \mathrm{~h}$ after one-lung ventilation and postoperative $24 \mathrm{~h}(P<.01)$. The MMSE score of the Dex group was significantly higher than the control group at postoperative 7 days and postoperative 30 days $(P<.01)$. Although with no statistical significance, the MMSE score of the Dex group was higher than the control group at postoperative 90 days $(P>.05)$. The incidence of postoperative cognitive dysfunction (POCD) in the Dex group was significantly lower than that in the control group at postoperative 7 days and postoperative 30 days $(P<.05)$.

Conclusion: Dex used in elderly patients undergoing minimally invasive coronary artery bypass graft surgery can effectively increase the expression level of $\mathrm{Ngb}$ and reduce the incidence of POCD.

\section{INTRODUCTION}

Postoperative cognitive dysfunction (POCD) refers to the condition of neurocognitive decline following surgery in a cognitive and sensory manner [Evered 2018]. POCD has been considered in the anesthetic and surgical literature in isolation

Received fune 22, 2021; received in revised form fuly 29,2021; accepted fuly 30, 2021.

Correspondence: Finliang Teng, Department of Anesthesiology, The First Affiliated Hospital of Hebei North University, No.12, Changqing Street, Qiaoxi District, Zhangiakou, Hebei 075000, China; +86-0313-8046933 (e-mail: tengjinliang@126.com). of cognitive decline, which is common in the elderly within the community and where it is labeled as mild cognitive impairment, neurocognitive disorder, or dementia [Yazit 2020].

Dexmedetomidine (Dex) is a highly selective $\alpha 2$ receptor agonist with analgesic, sedative, and anti-inflammatory effects that has been shown to reduce the incidence of POCD [Mohite 2019]. Minimally invasive coronary artery bypass graft surgery requires single-lung ventilation, which can cause hypoxemia and affect postoperative cognitive function [Kammerer 2016; Lopez 2017].

Neuroglobin $(\mathrm{Ngb})$ is a globulin that exists in vertebrates and is abundantly expressed in neurons [Gao 2020; Zhang 2019]. It has a high affinity for oxygen, thus increasing the oxygen supply to brain tissue [Zhu 2017]. Ngb is a recently discovered hypoxia-inducible protein, which has a cytoprotective effect in animal models of Alzheimer's disease, stroke, myocardial infarction, and other related diseases [Khan 2006]. Therefore, the purpose of this study was to observe the effect of Dex on the expression of $\mathrm{Ngb}$ and postoperative cognitive function in elderly patients with minimally invasive coronary artery bypass graft surgery and to provide clinical references.

\section{MATERIALS AND METHODS}

\section{Patients}

Forty patients, who underwent elective minimally invasive off-pump coronary artery bypass grafting (OPCABG) in our hospital from January 2018 to December 2019, were enrolled. Patients were aged $65-77$ years, with weight of $56-75 \mathrm{~kg}$.

Inclusion criteria were patients with 1) ASA II or III, and 2) HYHA II or III. Exclusion criteria were patients with 1) dementia and mental history, 2) severe cerebrovascular disease, 3) history of multiple operations, 4) history of severe infection of various systems during the perioperative period, 5) severe perioperative blood loss, severe acid-base balance and electrolyte disturbance, 6) severe abnormal liver and kidney function, 7) no acute myocardial infarction, 8) no history of brain trauma or cerebrovascular accident, 9) no history of diabetes, and 10) no history of mental illness.

With random number tables, the patients were randomly divided into the Dex group $(\mathrm{N}=20)$ and control group $(\mathrm{N}=$ 20). This study was approved by the Medical Ethics Committee of the First Affiliated Hospital of Hebei North University (No. K2017001), and all patients signed informed consent forms. 
Table 1. General intraoperative information of the two groups

\begin{tabular}{lccc}
\hline & $\begin{array}{c}\text { Dex group } \\
(\mathrm{N}=20)\end{array}$ & $\begin{array}{c}\text { Control group } \\
(\mathrm{N}=20)\end{array}$ & $P$ \\
\hline Male, n (\%) & $9(45)$ & $10(50)$ & .752 \\
Age, years & $70.5 \pm 4.1$ & $71.4 \pm 4.5$ & .513 \\
Height & $166.2 \pm 5.3$ & $167.3 \pm 5.4$ & .518 \\
Weight (kg) & $68.3 \pm 10.1$ & $69.1 \pm 9.6$ & .811 \\
ASA grades, n (\%) & - & - & .752 \\
II & $10(50)$ & $11(55)$ & \\
III & $10(50)$ & $9(45)$ & \\
HYHA grades, n (\%) & - & - & .749 \\
II & $8(40)$ & $9(45)$ & \\
III & $12(60)$ & $11(55)$ & \\
Number of bypass grafts, $\mathrm{n}(\%)$ & $1(5)$ & $1(5)$ & \\
Hypertension, n (\%) & $15(75)$ & $14(70)$ & .732 \\
\hline
\end{tabular}

Age, height, and weight were compared using independent sample t-test. Gender, ASA grades, HYHA grades, and number of bypass grafts were compared using $\chi^{2}$ test.

Table 2. Incidence of POCD in the two groups

\begin{tabular}{lccc}
\hline & $\begin{array}{c}\text { Dex group } \\
(\mathrm{N}=20)\end{array}$ & $\begin{array}{c}\text { Control group } \\
(\mathrm{N}=20)\end{array}$ & $P$ \\
\hline Postoperative 7 days & $2(10)$ & $8(40)$ & .028 \\
Postoperative 30 days & 0 & $4(20)$ & .027 \\
Postoperative 90 days & 0 & $2(10)$ & .468 \\
\hline
\end{tabular}

POCD, postoperative cognitive dysfunction. Incidence of POCD was compared using $\chi^{2}$ test.

\section{Anesthesia method}

The two groups of patients routinely abstained from drinking and fasted for 8 hours before surgery, and no preoperative medication was used. NIBP, ECG and SpO2 were monitored continuously after entry. The left radial artery was punctured and catheterized under local anesthesia to monitor arterial blood pressure. Anesthesia induction: midazolam $0.04 \mathrm{mg} / \mathrm{kg}$, sufentanil $3 \mu \mathrm{g} / \mathrm{kg}$, etomidate $0.2 \mathrm{mg} / \mathrm{kg}$, cisatracurium $0.3 \mathrm{mg} / \mathrm{kg}$.

After induction, double-lumen endobronchial intubation was performed. During bilateral lung ventilation, tidal volume VT was $7 \sim 8 \mathrm{~mL} / \mathrm{kg}$, respiratory rate RR was $10-12$ beats $/ \mathrm{min}$, and inspiratory-expiratory ratio I:E=1:2. During one-lung ventilation, $\mathrm{VT}=6 \mathrm{~mL} / \mathrm{kg}, \mathrm{RR}=12-16$ times $/ \mathrm{min}$, other ventilation parameters remain unchanged, and PETCO2 is maintained at $35-45 \mathrm{mmHg}$. A four-cavity central venous catheter was inserted into the right internal jugular vein puncture for fluid infusion, CVP monitoring and pumping of vasoactive
Table 3. The hospital stay and postoperative delirium rate in the two groups

\begin{tabular}{lccc}
\hline & $\begin{array}{c}\text { Dex group } \\
(\mathrm{N}=20)\end{array}$ & $\begin{array}{c}\text { Control group } \\
(\mathrm{N}=20)\end{array}$ & $P$ \\
\hline ICU stay (h) & $35 \pm 11$ & $46 \pm 12$ & $<.001$ \\
Hospital stay (d) & $10 \pm 1$ & $13 \pm 2$ & $<.001$ \\
Postoperative 7 days & $3(15)$ & $10(50)$ & $<.001$ \\
Postoperative 30 days & 0 & $5(25)$ & $<.001$ \\
Postoperative 90 days & 0 & $2(10)$ & .468 \\
\hline
\end{tabular}

ICU stay and hospital stay were compared using independent sample t-test. Postoperative delirium rate was compared using $\chi^{2}$ test.

Table 4. Comparison of $\mathrm{Ngb}$ between the two groups

\begin{tabular}{lccc}
\hline & $\begin{array}{c}\text { Dex group } \\
(\mathrm{N}=20)\end{array}$ & $\begin{array}{c}\text { Control group } \\
(\mathrm{N}=20)\end{array}$ & $P$ \\
\hline Preoperative 24h $(\mathrm{ng} / \mathrm{ml})$ & $0.15 \pm 0.03$ & $0.16 \pm 0.02$ & .08 \\
6h after one-lung ventilation $(\mathrm{ng} / \mathrm{ml})$ & $0.28 \pm 0.04 *$ & $0.19 \pm 0.04$ & $<.001$ \\
Postoperative 24h $(\mathrm{ng} / \mathrm{ml})$ & $0.25 \pm 0.05^{*}$ & $0.17 \pm 0.03$ & $<.001$ \\
\hline
\end{tabular}

$*<0.01$, compared with preoperative $24 \mathrm{~h}$. Ngb, neuroglobin. Ngb was compared using independent sample t-test.

drugs. $4-6 \mathrm{mg} /(\mathrm{kg} \cdot \mathrm{h})$ of propofol and $0.8-1 \mu \mathrm{g} /(\mathrm{kg} \cdot \mathrm{h})$ of sufentanil were continuously infused for anesthesia maintenance.

According to the length of operation, $10 \mathrm{mg}$ cis-atracurium was injected intermittently to maintain BIS at 40-60. Patients in the Dex group were injected with a loading dose of $0.6 \mu \mathrm{g} /$ $\mathrm{kg}$ Dex within 15 minutes before induction of anesthesia, followed by continuous pump injection at a rate of $0.2 \mu \mathrm{g} /(\mathrm{kg} \cdot \mathrm{h})$ until the end of the operation; the control group was given the same dose of $0.9 \%$ normal saline.

Intraoperative infusion of Ringer's lactate solution, voluven, and autologous blood was performed according to the situation, and vasoactive drugs were used to maintain stable circulation when necessary. At the end of the operation, the infusion of anesthesia was stopped, and the tracheal catheter was returned to the ICU ward. All operations were performed by the same surgeon. After the patient woke up, the postoperative analgesia pump (formula: sufentanil $200 \mu \mathrm{g}+$ tolansetron $4 \mathrm{mg}+$ normal saline $100 \mathrm{ml}$ ) was administered at $2 \mathrm{ml} / \mathrm{h}$ for continuous analgesia for $50 \mathrm{~h}$.

\section{Observation indexes}

The primary endpoint is the incidence of POCD. The mini mental status examination (MMSE) was used to detect the cognitive function of patients in the two groups at preoperative 1 day, postoperative 7 days, postoperative 30 days and postoperative 90 days, and the criteria were as follows: a total score of 30, 27-30 as normal, and <27 as POCD [Yang 2020]. The 
Table 5. MMSE comparison of the two groups

\begin{tabular}{lccc}
\hline & $\begin{array}{c}\text { Dex group } \\
(\mathrm{N}=20)\end{array}$ & $\begin{array}{c}\text { Control group } \\
(\mathrm{N}=20)\end{array}$ & $P$ \\
\hline Preoperative 1 day & $28.5 \pm 1.4$ & $28.4 \pm 1.3$ & .745 \\
Postoperative 7 days & $24.8 \pm 1.3^{*}$ & $20.5 \pm 1.4^{*}$ & $<.001$ \\
Postoperative 30 days & $28.3 \pm 1.5 \dagger$ & $24.2 \pm 1.2^{*} \dagger$ & $<.001$ \\
Postoperative 90 days & $28.4 \pm 1.3 \dagger$ & $26.6 \pm 1.5 \dagger$ & $<.001$ \\
\hline
\end{tabular}

$*<0.01$, compared with preoperative 1 day; $\uparrow<0.01$, compared with postoperative 7 days. MMSE, mini mental status examination. MMSE was compared using independent sample t-test.

definition of delirium is based on the "Diagnostic and Statistical Manual of Mental Disorders" (DSM IV) [Association AP 1994] criteria: (1) consciousness disorder with reduced ability to focus, sustain, or shift attention; (2) cognitive changes or development of perceptual disorders; (3) acute onset and fluctuating course, and (4) evidence of organic ethological factors. Venous blood samples were taken preoperative $24 \mathrm{~h}, 6 \mathrm{~h}$ after one-lung ventilation, and postoperative $24 \mathrm{~h}$ to determine the expression level of serum Ngb in both groups. The incidence of postoperative complications was compared between the two groups.

\section{Statistical analysis}

The trial was powered for tests for two proportions of Dex against normal saline on the incidence of POCD. The sample size calculation was based on the assumption of the incidence of POCD of $0.01 \%$ in the Dex group and $16.70 \%$ in the control group [Gao 2020]. We estimated that the assignment of 40 patients in a 1:1 ratio to the Dex group versus the control group would provide a power of $64 \%$ with a one-sided alpha level of 0.10 .

All analyses were performed using SPSS 20.0 software (SPSS, Inc., Chicago, IL, USA). Quantitative data were described as mean \pm standard deviation (SD). Normally distributed data, including age, height, weight, ICU stay, hospital stay, Ngb, and MMSE were compared using independent sample t-test. Categorical variables, including gender, ASA grades, HYHA grades, number of bypass grafts, incidence of POCD and postoperative complications were presented as numbers (percentage) and compared using $\chi^{2}$ test. Two-tailed probability value of $P<.05$ was considered as statistically significant.

\section{RESULTS}

There were no significant differences in the general intraoperative information, including gender, age, height, weight, ASA grades, HYHA grades, operation time, number of bypass grafts, and hypertension $(P>.05)($ Table 1$)$.

\section{POCD}

The incidence of POCD in the Dex group was significantly lower than that in the control group at postoperative 7 days and postoperative 30 days $(P<.05)$ (Table 2$)$.
Table 6. The incidence of postoperative complications in the two groups

\begin{tabular}{lccc}
\hline & $\begin{array}{c}\text { Dex group } \\
(\mathrm{N}=20)\end{array}$ & $\begin{array}{c}\text { Control group } \\
(\mathrm{N}=20)\end{array}$ & $P$ \\
\hline Incision infection & $1(5)$ & $2(10)$ & $>.999$ \\
Ventricular fibrillation & 0 & 0 & $>.999$ \\
Respiratory complications & $4(20)$ & $5(25)$ & $>.999$ \\
Transient renal insufficiency & $1(5)$ & $1(5)$ & $>.999$ \\
\hline
\end{tabular}

Postoperative complications were compared using $\chi^{2}$ test.

\section{Postoperative delirium rate}

The ICU stay and hospital stay in the Dex group was significantly lower than the control group. The postoperative delirium rates in the Dex group were significantly lower than that in the control group at postoperative 7 days and postoperative 30 days $(P<.05)$ (Table 3$)$.

$\mathrm{Ng} b$

The expression level of Ngb in the Dex group was significantly higher than that in the control group at $6 \mathrm{~h}$ after onelung ventilation and postoperative $24 \mathrm{~h}(P<.01)$. The expression level of $\mathrm{Ngb}$ at preoperative $24 \mathrm{~h}$ was significantly lower than that at $6 \mathrm{~h}$ after one-lung ventilation and postoperative $24 \mathrm{~h}$ in the Dex group $(P<0.01)$ (Table 4$)$.

\section{MMSE score}

The MMSE score of Dex group at postoperative 7 days was significantly lower than that at preoperative 1 day $(P<.01)$, but the MMSE score at postoperative 30 days and postoperative 90 days were significantly higher than that at postoperative 7 days $(P<.01)$. The MMSE score of the Dex group was significantly higher than the control group at postoperative 7 days and postoperative 30 days $(P<.01)$. Although with no statistical significance, the MMSE score of the Dex group was higher than the control group at postoperative 90 days $(P>.05)$ (Table 5).

\section{Postoperative complications}

There were no significant differences in the incidence of postoperative complications between the two groups $(P>.05)$ (Table 6).

\section{DISCUSSION}

Minimally invasive surgery has the advantage of small trauma and quick recovery, which is favored by patients with coronary heart disease. However, bypass of the anterior descending and diagonal branches is suitable for beatingheart coronary artery bypass surgery due to the limited surgical field. In this study, all 40 patients underwent anterior descending coronary artery bypass graft. Beating-heart coronary artery bypass surgery requires single-lung ventilation 
[Saha 2019], which is prone to decrease blood oxygen saturation during operation, and its decrease is closely related to the occurrence of POCD [Lopez 2017].

The incidence of POCD in the first week after cardiac surgery has been reported to be as high as $30-60 \%$, which may prolong the length of hospital stay, reduce the quality of life, increase postoperative mortality, and cause a serious burden on individuals and society [Mashour 2015]. Ngb is an oxygen-binding protein that is mainly expressed in nervous tissues where it is considered to be neuroprotective during ischemic brain injury. Therefore, we investigated the relationship between the occurrence of cognitive dysfunction and the expression level of $\mathrm{Ngb}$ after minimally invasive coronary artery bypass graft surgery.

Dex can reduce isoflurane-induced neurocognitive damage, acetaminophen-induced liver damage, and myocardial and liver ischemia-reperfusion through a variety of signaling pathways injury [Chou 2019; Geng 2019], it has been clinically proven that Dex can reduce the incidence of delirium and is a better choice for elderly critically ill patients.

Studies have found that Dex can up-regulate the expression level of $\mathrm{Ngb}$ and improve the long-term learning and memory ability of rats after hypoxia/reoxygenation injury in the developmental period [Gao 2019]. The results of this study showed that the Ngb expression level of the Dex group significantly increased at $6 \mathrm{~h}$ after one-lung ventilation and 24h after surgery, while the Ngb expression was not significantly upregulated in the control group, and the Ngb expression level of the Dex group was significantly higher than that of the control group. In addition, the incidence of POCD in the Dex group decreased significantly at postoperative 7 days and 30 days, suggesting that the improvement of POCD by Dex may be related to the increase of $\mathrm{Ngb}$ expression level.

MMSE is a POCD screening scale that is simple to operate and has good sensitivity. It is suitable for repeated assessment of cognitive status over time and can reflect the cognitive function status of patients [Myrberg 2020]. Studies have shown that in elderly patients undergoing cardiac surgery, postoperative intravenous acetaminophen combined with intravenous injection of propofol or Dex can reduce postoperative delirium [Subramaniam 2019]. Xu et al [Xu 2017] found that Dex can improve the postoperative cognitive function of elderly patients undergoing laparoscopic ovarian cystectomy under general anesthesia, and effectively reduce the incidence of POCD without obvious or serious adverse reactions. Similarly, the cognitive function status of the control group was significantly lower at postoperative 7 days and 30 days than that before operation, which improved at postoperative 90 days, while Dex can significantly improve the cognitive function at postoperative 7 days and 30 days and has a good preventive effect on the occurrence of postoperative dysfunction in elderly patients.

The limitation of this study was the small sample size, since our hospital is a non-cardiac hospital, and the conclusions remain to be verified by a large randomized controlled study.

\section{CONCLUSION}

Dex used in elderly patients undergoing minimally invasive coronary artery bypass graft surgery can effectively increase the expression level of $\mathrm{Ngb}$ and reduce the incidence of POCD.

\section{ACKNOWLEDGEMENT}

Funding: The study was supported by 2019 Medical Science Research Project of Hebei Provincial Health Commission (20190890).

\section{REFERENCES}

Association AP. 1994. American Psychiatric Association: Diagnostic and Statistical Manual of Mental Disorders. In Washington, DC. American Psychiatric Press.

Chou AH, Liao CC, Lee HC, et al. 2019. The MAP2K4/JNK/c-Jun Signaling Pathway Plays A Key Role In Dexmedetomidine Protection Against Acetaminophen-Induced Liver Toxicity. Drug Des Devel Ther. $13: 3887-98$

Evered LA, Silbert BS. 2018. Postoperative Cognitive Dysfunction and Noncardiac Surgery. Anesth Analg. 127:496-505.

Gao Y, Yin H, Zhang Y, et al. 2019. Dexmedetomidine protects hippocampal neurons against hypoxia/reoxygenation-induced apoptosis through activation HIF-1 $\alpha /$ p 53 signaling. Life Sci. 232:116611.

Gao Y, Zhang Y, Dong Y, et al. 2020. Dexmedetomidine Mediates Neuroglobin Up-Regulation and Alleviates the Hypoxia/Reoxygenation Injury by Inhibiting Neuronal Apoptosis in Developing Rats. Front Pharmacol. 11:555532.

Gao Y, Zhu X, Huang L, et al. 2020. Effects of dexmedetomidine on cerebral oxygen saturation and postoperative cognitive function in elderly patients undergoing minimally invasive coronary artery bypass surgery. Clin Hemorheol Microcirc. 74:383-9.

Geng Y, Li R, He SX, et al. 2019. Dexmedetomidine Attenuates Acute Lung Injury Induced by Heatstroke and Improve Outcome. Shock. 52:532-9.

Kammerer T, Speck E, von Dossow V. 2016. [Anesthesia in thoracic surgery]. Anaesthesist. 65:397-412.

Khan AA, Wang Y, Sun Y, et al. 2006. Neuroglobin-overexpressing transgenic mice are resistant to cerebral and myocardial ischemia. Proc Natl Acad Sci U S A. 103:17944-8.

Lopez MG, Pandharipande P, Morse J, et al. 2017. Intraoperative cerebral oxygenation, oxidative injury, and delirium following cardiac surgery. Free Radic Biol Med. 103:192-8.

Mashour GA, Woodrum DT, Avidan MS. 2015. Neurological complications of surgery and anaesthesia. Br J Anaesth. 114:194-203.

Mohite V, Baliga S, Thosar N, et al. 2019. Role of dexmedetomidine in pediatric dental sedation. J Dent Anesth Pain Med. 19:83-90.

Myrberg K, Hydén LC, Samuelsson C. 2020. The mini-mental state examination (MMSE) from a language perspective: an analysis of test interaction. Clin Linguist Phon. 34:652-70.

Saha S, Varghese S, Herr M, et al. 2019. Minimally invasive versus 
conventional extracorporeal circulation circuits in patients undergoing coronary artery bypass surgery: a propensity-matched analysis. Perfusion. 34:590-7.

Subramaniam B, Shankar P, Shaefi S, et al. 2019. Effect of Intravenous Acetaminophen vs Placebo Combined With Propofol or Dexmedetomidine on Postoperative Delirium Among Older Patients Following Cardiac Surgery: The DEXACET Randomized Clinical Trial. Jama. 321:686-96.

Xu HY, Fu GH, Wu GS. 2017. Effect of dexmedetomidine-induced anesthesia on the postoperative cognitive function of elder patients after laparoscopic ovarian cystectomy. Saudi J Biol Sci. 24:1771-5.

Yang Q, Cao J, Sun J. 2020. Effects of different doses of dexmedetomidine on cognitive function and stress response in patients undergoing cardiopulmonary bypass surgery Zhejiang Medical Journal. v.42:83-7.

Yazit NAA, Juliana N, Das S, et al. 2020. Association of Micro RNA and Postoperative Cognitive Dysfunction: A Review. Mini Rev Med Chem. 20:1781-90.

Zhang Y, Yang F, Gao Y, et al. 2019. Neuroglobin protects offspring rats from neuronal damage induced by sevoflurane exposure to pregnant rats by inhibiting endogenous apoptosis. Int J Dev Neurosci. 76:17-24.

Zhu L, Huang L, Wen Q, et al. 2017. Recombinant human erythropoietin offers neuroprotection through inducing endogenous erythropoietin receptor and neuroglobin in a neonatal rat model of periventricular white matter damage. Neurosci Lett. 650:12-7. 